\title{
Early developmental outcomes in children following convulsive status epilepticus: A longitudinal study
}

\author{
$*$ Marina M. Martinos, $\uparrow$ Michael Yoong, $†$ Shekhar Patil, $\$ W$ ui K. Chong, $\$$ Rodica Mardari, \\ $\dagger \S \rrbracket$ Richard F. M. Chin, $\uparrow q B$ rian G. R. Neville, *Michelle de Haan, and $\uparrow q \#$ Rod C. Scott \\ *Developmental Cognitive Neurosciences Unit, UCL Institute of Child Health, London, United Kingdom; $†$ Neurosciences and Mental
Health Unit, UCL Institute of Child Health, London, United Kingdom; $¥$ Department of Radiology, Great Ormond Street Hospital,
London, United Kingdom; §The Muir Maxwell Epilepsy Centre, University of Edinburgh, Edinburgh, United Kingdom;
ๆYoung Epilepsy, Lingfield, Surrey, United Kingdom; and \#Geisel School of Medicine at Dartmouth, Lebanon, New Hampshire, U.S.A.
}

\section{SUMMARY}

Purpose: Convulsive status epilepticus (CSE) is the most common pediatric neurologic emergency and is often associated with unfavorable neurodevelopmental outcomes. The early developmental trajectory of children following CSE has not been previously investigated, leaving a gap in our understanding of how these adverse longterm outcomes emerge.

Methods: We prospectively recruited children aged between $I$ and $\mathbf{4 2}$ months from a predefined geographic region of North London who had at least one episode of CSE and classified them as prolonged febrile seizures (PFS) or nonfebrile CSE. Neuropsychological and imaging investigations were conducted within 6 weeks of CSE (baseline) and were repeated a year later (follow-up). Neurodevelopment was assessed using the Bayley Scales of Infant Development III and compared to normally developing children. Predictors of neurodevelopmental scores at baseline and follow-up were investigated using regression analyses.

Key Findings: Of the 54 children that underwent investigations a mean of 38 days following CSE, 27 had PFS (mean age 18.4 months) and 27 had nonfebrile CSE (mean age I 5.5 months). In addition, I 7 healthy controls were assessed (mean age 20.49 months). Children with nonfebrile CSE had a worse developmental outcome than children with PFS $(p<0.002)$, despite there being no differences in seizure characteristics. In contrast to expectations, the PFS group had a worse developmental outcome than controls $(p=\mathbf{0 . 0 0 2})$. There were no significant differences in performance from baseline to I-year follow-up for the $\mathbf{7 0 . 4 \%}$ of children who provided data. Seizure characteristics were not shown to be significant predictors of performance.

Significance: CSE is associated with developmental impairments within 6 weeks of the acute event that continue to be present a year onward. This is also true of PFS cases that under-perform relative to controls despite mean scores within the clinically normal range. The absence of a change in performance from baseline to follow-up as well as the lack of a relationship between seizure characteristics and developmental outcomes supports the notion that premorbid abilities may be overshadowing any direct effects of CSE itself on outcome.

KEY WORDS: Prolonged febrile seizures, Developmental outcomes, Cognition, Language.
Convulsive status epilepticus (CSE), the most common pediatric neurologic emergency (DeLorenzo et al., 1995), is associated with significant mortality and morbidity (Raspall-Chaure et al., 2006). The incidence of CSE is highest within the first 3 years of life (Chin et al., 2006), a period during which critical developmental events occur (Committee on Integrating the Science of Child Development, 2000). Early neurologic insults are associated with worse longer term outcomes in retrospective studies (Riney

Accepted January 28, 2013; Early View publication April 08, 2013.

Address correspondence to Marina M. Martinos, Developmental Cognitive Neuroscience Unit, 30 Guilford Street, London WC1N 1EH, U.K. E-mail:m.martinos@ich.ucl.ac.uk

Wiley Periodicals, Inc.

(C) 2013 International League Against Epilepsy et al., 2006; Cormack et al., 2007; Scott, 2010), and animal studies have shown that enriching an animal's environment can modulate the degree of functional impairments following CSE in the longer term (Rutten et al., 2002). Therefore, it is imperative to assess the effects of CSE in children soon after CSE to determine their developmental status and examine whether intervention is warranted.

Most studies investigating the consequences of CSE to date: (1) have focused on simple febrile seizures and have included a subsample of children with prolonged febrile seizures (PFS), a type of CSE, within their study sample (Schiottz-Christensen \& Bruhn, 1973; Nelson \& Ellenberg, 1978; Hesdorffer et al., 2011), (2) have been conducted several years following CSE (Nelson \& Ellenberg, 1978; Verity et al., 1985, 1993; Kölfen et al., 1998), and (3) have been 
performed without the use of standardized neuropsychological instruments (Aicardi \& Chevrie, 1970; van Esch et al., 1996). The aims of the current study were the following: (1) to investigate cognitive, language, and motor functions in children within 6 weeks of CSE and a year later to determine their developmental trajectories; and (2) to identify risk factors for poor outcomes at baseline (within 6 weeks) and follow-up (1 year later). PFS, which occur in children with normal development, were investigated separately from those with nonfebrile CSE, who often have previous neurologic abnormalities.

\section{Methods}

\section{Participants}

We prospectively recruited children aged 1-42 months who had at least one episode of CSE between December 2006 and March 2010. Patients were recruited from north London hospitals using a recruitment strategy similar to the North London Convulsive Status Epilepticus Surveillance study (Chin et al., 2006), which is described in detail elsewhere (Yoong et al., 2012). In brief, we collected baseline clinical data and arranged for the child to have a magnetic resonance imaging (MRI) scan and neuropsychological assessments at University College London Institute of Child Health (ICH)/Great Ormond Street Hospital (GOSH) at the parents' earliest convenience. When patients attended GOSH for their brain MRI, a clinical history was taken by MY from each family, which included the following: medical history; seizure semiology; and any concerns about development prior to CSE. Participating families in our patient population were invited back a year later for repeat assessments.

We also recruited control participants of a similar age range in whom parents or caregivers had no concerns about development prior to the assessment and who had no previous history of provoked or unprovoked seizures. Control participants were recruited via parenting groups, cinema screenings for mothers and their babies, and through acquaintances with children in that age range. Dizygotic twin siblings of two PFS infants were also assessed, and their results are reported separately from those of other control participants. Control participants were only seen once during our study. Indices of multiple deprivation (IMDs) were calculated for all participants (http://www.ons.gov.uk) and used as proxy measure for individual socioeconomic status (SES). IMD scores are calculated on the basis of home address postal codes and comprise seven separate dimensions of deprivation (income, employment, health, education, housing, crime, and living environment) the individual scores of which have been standardized and combined in a weighted manner to make a single score for the smallest socially homogenous local area. Increasing scores correlate with worsening SES (Office of the Deputy Prime Minister, 2007).

\section{Operational definitions}

CSE was defined as a tonic, clonic, or tonic-clonic seizure (continuous), or two or more seizures between which consciousness was not regained (intermittent), which lasted for at least $30 \mathrm{~min}$. Seizure duration was estimated from the initial referral report and corroborated by parental recollection. Two pediatric neurologists (RCS and RFMC) independently reviewed each case and classified the children into two groups: a PFS group and a nonfebrile CSE group. PFS was defined as CSE in an otherwise neurologically normal child from the age of 6 months with a fever (temperature of $\geq 38^{\circ} \mathrm{C}$ ) (International League Against Epilepsy, 1993). The nonfebrile CSE group included the following five etiologic groups as defined in our previous studies (Chin et al., 2006): (1) acute symptomatic (AS), (2) remote symptomatic (RS), (3) idiopathic epilepsy related (IER), (4) cryptogenic epilepsy related (CER), and, (5) unclassified CSE (UN). Any differences in opinion were resolved by consensus discussion.

\section{Neurodevelopmental assessments}

A neuropsychologist (MM) blinded to medical details and patient classification carried out the assessments. Infants were assessed using the Bayley Scales of Infant and Toddler Development (3rd edition; Bayley, 2005). The Bayley scales provide three normative composites with a mean of a 100 and a standard deviation of 15: cognitive, language and motor composites. When necessary, the Bayley composite scores were corrected for prematurity.

\section{Magnetic resonance imaging}

MRI investigations were carried out in a Siemens Avanto $1.5 \mathrm{~T}$ (Erlangen, Germany) whole body system using standard epilepsy protocol imaging. The images were reviewed by two experienced pediatric neuroradiologists (WKC and $\mathrm{RG}$ ) blinded to the patient's medical history who agreed by consensus whether the scan contained no abnormalities, minor abnormalities, or major abnormalities. A minor abnormality was defined as an abnormal feature thought to be either unrelated to this episode of CSE or of no functional significance. A major abnormality was defined as an abnormal feature likely to have significant effect on the child and/or represent a cause for this episode of CSE.

\section{Ethics}

The study was approved by the ICH/GOSH Research Ethics Committee. Local Research and Development registration was obtained at all referring hospitals. Written informed consent to participate in the study was obtained from all parents at the initial assessment.

\section{Statistical analyses}

Analysis was done with Predictive Analytics Software (PASW) version 18 (Chicago, IL, U.S.A.) for Windows. A p-value of 0.05 was used as the cut-off point for statistical 
significance. We defined our first assessment post CSE as our baseline assessment. Comparisons of group characteristics were made using chi-square analyses, independent sample $t$-tests, and one way analysis of variance (ANOVA). To reduce our dataset, we ran a principal component analysis (PCA) on the Bayley composites that derived one factor with an eigenvalue larger than 1 . We subsequently ran a univariate ANOVA on the derived factor to detect any differences in performance between the PFS, the nonfebrile CSE, and the control groups, controlling for age in months, gender, prematurity ( $<36$ weeks of gestation), English as a first language (yes or no), and SES.

To investigate our primary hypothesis, that is, that seizure characteristics per se predict outcomes after CSE, we ran a univariate ANOVA on the PCA-derived factor for the two patient groups combined. Seizure duration, days elapsed from CSE, focality of seizure (generalized or focal), and type of seizure (continuous or intermittent) were included in our model. To investigate our secondary hypothesis, that is, that prior neurologic insults predict outcomes after CSE we ran a univariate ANOVA on the PCA-derived factors for the two patient groups combined where we included age at assessment, prematurity, previous occurrence of CSE (yes or no), previous occurrence of short seizures (yes or no), and MRI abnormalities (normal, minor, major),. For the follow-up data, paired sample $t$-tests were carried out to measure significant changes in performance from baseline to follow-up. Pearson's correlations were conducted to investigate relationships between baseline and follow-up composites. Finally, regression analyses were performed to determine predictors of neurodevelopmental outcome in our two patient groups. Bootstrapping based on 1,000 samples was applied to all our analyses.

\section{RESULTS}

\section{Baseline}

One hundred fifty-one infants were referred during our recruitment period. From these, 54 parents agreed to take part in the study. The remaining CSE patients were not assessed for one of the following reasons: (1) families were noncontactable ( $\mathrm{N}=25)$, (2) patients were not suitable for sedation $(\mathrm{N}=10)$, (3) parents declined participation $(\mathrm{N}=43)$, (4) families lived far from the study center $(\mathrm{N}=10),(5)$ patients died prior to recruitment $(\mathrm{N}=7),(6)$ one family agreed to participate but never attended $(\mathrm{N}=1)$, and (7) one family only spoke Tamil $(\mathrm{N}=1)$. No differences in age $(p=0.478)$, gender $(p=0.600)$, SES $(p=0.759)$, or duration of seizure $(p=0.146)$ were present between the participating and the nonparticipating sample. However, the distribution of causes within the nonfebrile CSE group was found to be significantly different between the participating and the nonparticipating samples (cause was identifiable in 84 out of 97 nonparticipants; $\chi^{2}=14.65$, $\mathrm{p}=0.012$ ). This difference was driven by a larger proportion of acute symptomatic cases in the nonparticipating sample.

Table 1 provides the clinical and demographic characteristics of the study population. Children were assessed a mean of 38 days post CSE (mean duration $75.4 \mathrm{~min}$ ). Twentyseven infants had PFS and 27 children had nonfebrile CSE. Three children with a confirmed SCN1A mutation were included in the nonfebrile CSE group as their CSE was preceded by afebrile seizures. There were no differences between the two patient groups in seizure characteristics. These included age at CSE $(p=0.203)$, duration $(\mathrm{p}=0.207)$, time from seizure $(\mathrm{p}=0.180)$, proportion of

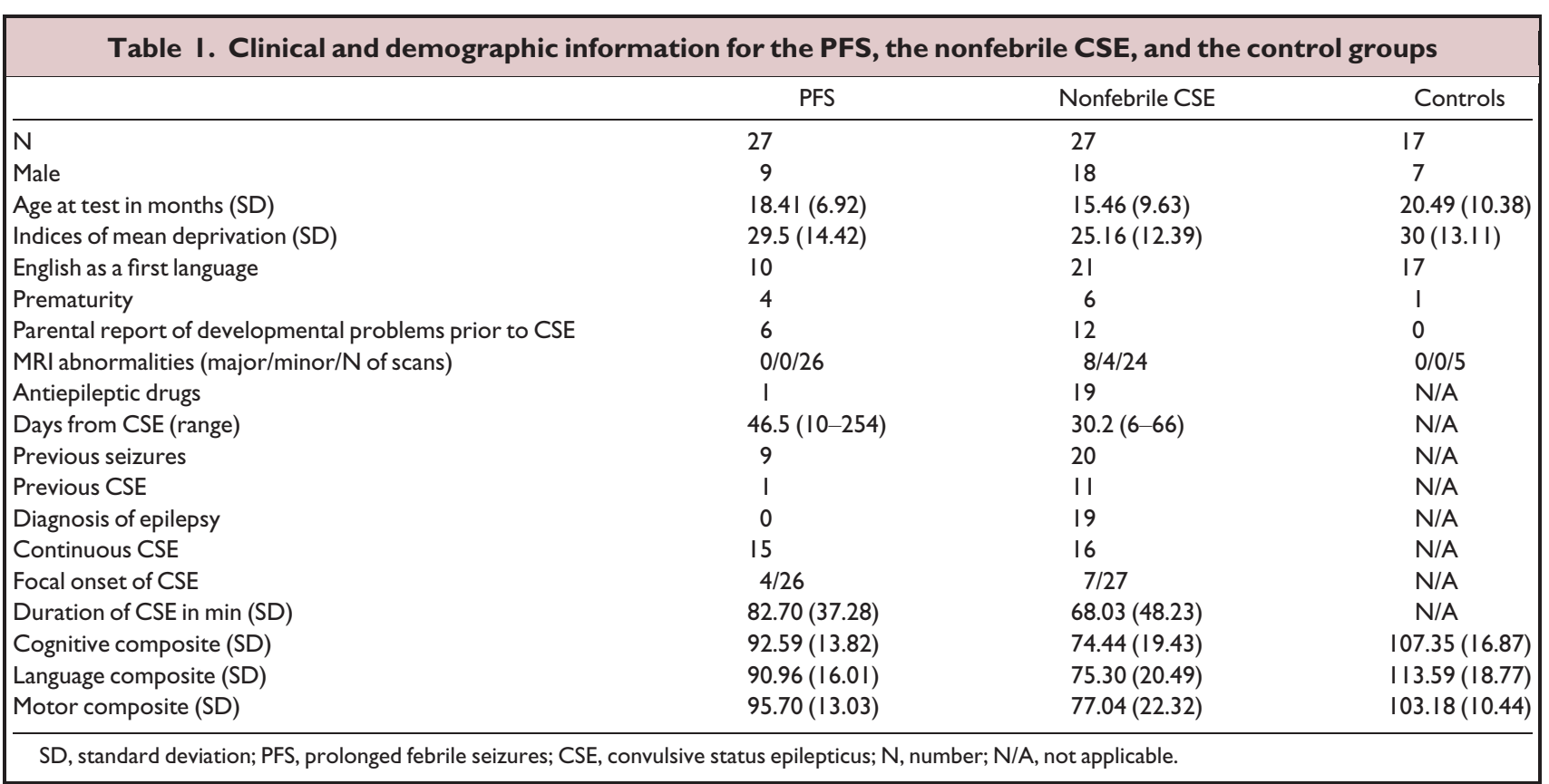


generalized versus focal onset of CSE $(p=0.321)$, and proportion of continuous versus intermittent CSE $(p=1)$. Proportion of developmental concerns prior to CSE was similar in both patient groups $(\mathrm{p}=0.232)$. Nevertheless, the type of developmental delay reported for the two groups was dissimilar in the severity of the reported symptoms, with the most frequently reported symptom in the PFS group being that of speech delay. Table S1 lists the kinds of developmental delay reported by parents as well as the results from our neuropsychological assessments for each of these cases.

Children with nonfebrile CSE were more likely to have an MRI abnormality with no visibly apparent MRI abnormality on any of the PFS scans ( $p<0.001$; see Table S2). In addition, children in the nonfebrile CSE group were far more likely to be on antiepileptic medication at the time of assessment, with only one child from the PFS group being on medication at the time of our baseline assessment $(p<0.001$; see Table S3). Finally, children with nonfebrile CSE were far more likely than children with PFS to have experienced CSE $(\mathrm{p}=0.001)$ or seizures $(\mathrm{p}=0.003)$ previously. Seizures experienced by the PFS group prior to recruitment were short febrile seizures, and only one child had experienced a previous PFS.

Seventeen healthy controls were assessed on the Bayley's developmental assessment. The PFS, nonfebrile CSE and the control groups were found to contain similar proportions of preterms $\left(\chi^{2}=4.18, \mathrm{p}=0.354\right)$ and did not significantly differ on SES $(F(2,67)=0.96, p=0.39)$. However, there were fewer participants with English as their first language $\left(\chi^{2}=18.62, \mathrm{p}<0.001\right)$ and fewer males $\left(\chi^{2}=6.65\right.$, $\mathrm{p}=0.036)$ within the PFS group compared to the other two groups.

\section{Are the patient groups different from controls?}

The PCA on the cognitive, language, and motor composite scores extracted one factor, which accounted for $83.25 \%$ of the total variance. The univariate ANOVA revealed main effects of group $(\mathrm{F}(2,68)=6.524, \quad \mathrm{p}=0.003)$, age $(\mathrm{F}(1,68)=9.294, \mathrm{p}=0.004)$, and prematurity $(\mathrm{F}(1,68)$ $=9.167, \mathrm{p}=0.004)$. Post hoc tests with Bonferroni correction for multiple comparisons revealed that both the nonfebrile CSE group $(p=0.001)$ and the PFS group $(p=0.002)$ performed below normal controls. In addition, the nonfebrile CSE group performed below the PFS group $(\mathrm{p}=0.002)$. In addition, we investigated whether the patients' scores differed from the normative mean for the Bayley's scales and confirmed the above results (Fig. 1).

In addition, both healthy twins outperformed their PFS siblings. One of the healthy twins performed better on all areas. The second healthy twin performed better in cognitive and motor scales but on a par with her PFS twin on the language scale. The difference in performance ranged from 3 to 25 points and was most pronounced in the cognitive scale (see Table S4).

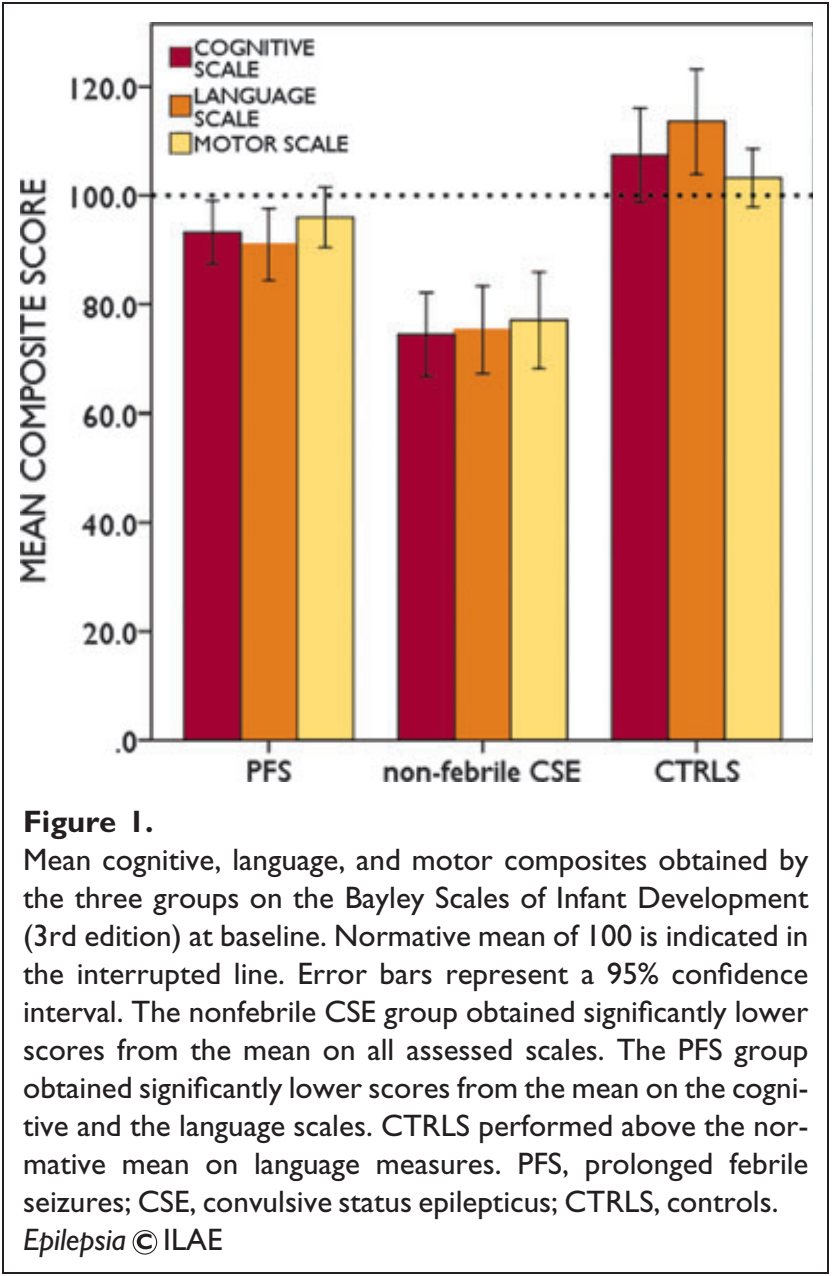

Pearson's correlations revealed a positive correlation between age and language performance in the PFS $(\mathrm{r}=0.572, \quad \mathrm{p}=0.003)$ and the control $(\mathrm{r}=0.647$, $\mathrm{p}=0.005)$ groups. No other areas of performance were found to be affected by age. The relationship between age and language performance was absent from the nonfebrile CSE group. Because this group includes a number of cases that scored at the bottom of the scale, such a relationship may be harder to identify. To determine whether this was the case we reran the correlation, excluding cases that scored lower than a 62 on the language scale, that is, the lowest score obtained by a PFS subject. There was still no correlation between age and language performance in this $\operatorname{group}(\mathrm{r}=0.221, \mathrm{p}=0.348)$.

\section{Which factors affect performance at baseline following CSE?}

The univariate ANOVA with the PCA-derived factor as the dependent variable and duration of CSE, type and focality of CSE, and days from CSE as the independent variables, revealed the absence of an effect of seizure-related variables 


\section{M. Martinos et al.}

on performance. On the contrary, our second ANOVA, which investigated the predictive value of neurologic abnormalities on performance, showed that presence of an abnormality on MRI $(\mathrm{F}(2,48)=3.489, \mathrm{p}=0.045)$, signs of developmental problems prior to CSE $(\mathrm{F}(1,48)=24.929$, $\mathrm{p}<0.001)$, and age at assessment $(\mathrm{F}(1,48)=6.614$, $\mathrm{p}=0.016)$ were significant predictors of outcome at baseline.

\section{Follow-up}

Thirty-eight children (70.4\% of the original sample) were seen for a follow-up assessment a mean of 12.6 months following their baseline assessment. From the remaining children, one died, one family moved outside the United Kingdom, two families became noncontactable, two children provided data that could not be converted into standardized scores, and ten families declined to participate in the follow-up stages of this study. Two children that had outgrown the Bayley scales at follow-up were assessed using the Wechsler Preschool and Primary Scale of Intelligence (WPPSI), which provided measures of their cognitive (i.e., full-scale intelligence quotient [FSIQ]) and language (i.e., verbal intelligence quotient [VIQ]) development but not of their motor development. Given that excluding their results made no difference to the outcome, their results were included in the current analysis.

Twenty-two children had been classified as PFS at baseline (81.5\% of the original sample) and 16 as nonfebrile CSE (59.6\% of the original sample); 7 as RS, 4 as CER, 3 as IER, and 2 as AS. Independent sample $t$-tests revealed no differences in demographic and clinical factors between the original and the follow-up samples. Moreover, there were no differences in the performance of the two groups at baseline on the cognitive, language, and motor scales, pointing to the representativeness of the follow-up sample. This was true even when the PFS and the nonfebrile CSE groups were investigated separately (Table 2).

Six of 22 children in the PFS group experienced short febrile seizures in the follow-up period, and one child had a PFS during that period. Fourteen of 16 children in the nonfebrile CSE group experienced seizures in the follow-up period. Three of these were CSE episodes. Finally, one child from the PFS group and 11 from the nonfebrile CSE group were on medication at follow-up.

\section{Are the groups performing differently at 1-year follow-up?}

Table 2 contains the means and standard deviations for the group's follow-up assessment. Paired sample $t$-tests revealed no difference in performance from baseline to follow-up for the PFS (cognition: $p=0.809$; language: $\mathrm{p}=0.181$, motor: $\mathrm{p}=0.394)$ and the nonfebrile CSE (cognition: $p=0.454$; language: $p=0.444$; motor: $\mathrm{p}=0.634$ ) groups.

In the PFS group, cognitive scores at baseline were positively correlated with cognitive scores at follow-up $(\mathrm{r}=0.585, \mathrm{p}=0.005)$. The same was true of language scores $(r=0.731, p<0.001)$ and motor scores $(r=0.612$, $\mathrm{p}=0.005)$. Linear regression revealed that age at the time of follow-up $(\mathrm{p}=0.002)$ as well as the baseline cognitive $(p=0.002)$ and baseline language $(p=0.048)$ composites were highly predictive of overall outcome (factor derived by PCA) at 12.4 months and were able to determine $87.7 \%$ of the total variance $(F(3,15)=28.653, p<0.001)$. Similarly, in the nonfebrile CSE group, baseline and follow-up cognitive $(r=0.713, p=0.002)$, language $(r=0.573$,

Table 2. Clinical and demographic information for follow-up participants and nonparticipants

\begin{tabular}{|c|c|c|c|c|}
\hline & $\begin{array}{c}\text { PFS } \\
\text { participants }\end{array}$ & $\begin{array}{c}\text { PFS } \\
\text { nonparticipants }\end{array}$ & $\begin{array}{l}\text { Nonfebrile } \\
\text { CSE participants }\end{array}$ & $\begin{array}{c}\text { Nonfebrile } \\
\text { CSE nonparticipants }\end{array}$ \\
\hline $\mathrm{N}$ & 22 & 5 & 16 & 11 \\
\hline Male & 8 & I & 12 & 6 \\
\hline Age at test in months (SD) & $18.09(6.8)$ & $19.86(8.08)$ & | $3.56(7.99)$ & $18.22(11.46)$ \\
\hline Indices of mean deprivation (SD) & $32(14.76)$ & I8.52 (4.84) & $25.55(10.49)$ & $24.59(15.28)$ \\
\hline English as a first language & 8 & 2 & 11 & 10 \\
\hline Prematurity & 3 & I & 2 & 4 \\
\hline Parental report of developmental problems prior to CSE & 3 & 3 & 7 & 5 \\
\hline MRI abnormalities (major/minor/N of scans) & 0 & 0 & $3 / 5 / 16$ & $1 / 3 / 8$ \\
\hline Antiepileptic drugs & I & 0 & II & 8 \\
\hline Previous seizures & 6 & 3 & 13 & 7 \\
\hline Previous CSE & 0 & 1 & 6 & 5 \\
\hline Continuous CSE & 12 & 4 & 10 & 6 \\
\hline Focal onset of CSE & 3 & I & 6 & I \\
\hline Duration of CSE in minutes (SD) & $81.95(30.48)$ & $86(64.36)$ & $72.19(59.19)$ & $62(26.94)$ \\
\hline Cognitive composite (SD) & $92.05(14.85)$ & $95(8.66)$ & $76.88(20.73)$ & $70.91(17.72)$ \\
\hline Language composite (SD) & $92.3(15.82)$ & $85.6(17.46)$ & $75.38(14.84)$ & 75.18 (27.59) \\
\hline Motor composite (SD) & $93.87(13.05)$ & $103.8(10.4)$ & $80.5(20.78)$ & $72(24.49)$ \\
\hline
\end{tabular}

SD, standard deviation; CSE, convulsive status epilepticus; N, number; PFS, prolonged febrile seizures; MRI, magnetic resonance imaging. 
$\mathrm{p}=0.020)$, and motor $(\mathrm{r}=0.652, \mathrm{p}=0.008)$ composites were positively correlated. Linear regression revealed that developmental outcome in the nonfebrile CSE group at follow-up was predicted by the baseline cognitive composite score $(\mathrm{p}<0.001)$ and whether the patient was on antiepileptic medication $(\mathrm{p}=0.002)$.

\section{Discussion}

This is the first study to report on the longitudinal neuropsychological outcomes of infants within 6 weeks of CSE and 1 year later. Our results show that following CSE, infants manifest a wide range of neurodevelopmental impairments that are present at both time points. It is important to note that these impairments are not only confined to the nonfebrile CSE cases, who are already associated with neurologic abnormalities at baseline, but are extended to the PFS cases, who perform worse than controls on the assessed measures. No significant differences were revealed between baseline and follow-up performance, thereby demonstrating that within the first year post CSE, developmental outcomes remain unchanged.

Our most unexpected finding was that children with PFS were shown to lag developmentally behind controls. Moreover, although, the PFS group was still found to perform within the clinically normal range, their scores were similar to those obtained by infants born very prematurely ( $<28$ weeks of gestation) and/or infants of an extremely low birth weight $(<1,000 \mathrm{~g}$; Anderson et al., 2010). These findings were paralleled by our observations in two sets of twins, where the affected twins had lower developmental scores than their siblings. In a separate study, we recently reported that children following PFS manifest recognition memory impairments within the first year following their seizure (Martinos et al., 2012).

A caveat to the above finding was that we present data only on children with PFS younger than 42 months as we wanted to use the same neuropsychological instrument for all participants. By definition, PFS occur from the age of 6 months to the age of 72 months. Nevertheless, most PFS occur before the age of 42 months (Chin et al., 2006) and, in the present study, the mean age of PFS was found to be 18.4 months, which is skewed to the lower end of that age range. The FEBSTAT study, which describes 199 cases following PFS, stated the mean age at PFS to be 16 months, with an interquartile range of 12-24 months (Hesdorffer et al., 2012) Therefore, the above reports suggest that the current findings represent most of the PFS population and are not excluding a sizeable chunk. Moreover, in our recognition memory paper where the same experimental task could be used for the entire age range, our results were not dependent upon the age of the child (Martinos et al., 2012).

Results in the literature are mixed with respect to the long-term outcome of children following PFS, with some studies reporting normal development (Nelson \& Ellenberg,
1978; Verity et al., 1993, 1998; Chang et al., 2000) and other studies reporting impairments (Schiottz-Christensen \& Bruhn, 1973; van Esch et al., 1996; Kölfen et al., 1998). However, such discrepancies may be due to differences in methodology and definition of normality across studies. For example, one study of monozygotic twins discrepant only for a history of febrile seizures found that the affected twin had a lower IQ than the unaffected twin (SchiottzChristensen \& Bruhn, 1973). Nevertheless, their IQ was still within the normal range. Moreover, studies have often used different durational cutoffs to each other, with some studies splitting their sample population into children that have seizures lasting less than and more than 15 min (e.g., Verity et al., 1998; Chang et al., 2000) and other studies using the 30-min durational cutoff adopted in the current study (e.g., Verity et al., 1993; van Esch et al., 1996). Given indications that seizures of the prolonged kind ( $>30 \mathrm{~min})$ are the ones that carry an association with mesial temporal sclerosis (Meldrum \& Brierley, 1973; Baram \& Shinnar, 2001), the present study was designed specifically to only investigate those type of seizures. It is notable, however, that recent evidence has revealed that expressive language deficits are present in a subset of children following short febrile seizures ( $<5 \mathrm{~min}$ ): children with recurrent febrile seizures (Visser et al., 2012).

There are at least three possible explanations for the reduced performance in the PFS group. The first is that PFS led to a brain injury that causes a cognitive lag. There is extensive evidence from animal models that CSE can cause injury, predominantly to the hippocampus, but also in other brain regions (Sankar et al., 1998; Ravizza et al., 2005; Choy et al., 2010). Moreover, long-term cognitive impairments has been identified in an animal model of PFS, where animals with previously normal brains were made to seize under high temperature conditions (Dubé et al., 2006, 2009). The second is that the factors that predispose an individual to develop PFS may also be responsible for the reduced performance we observed in the present study. Hippocampal abnormalities in unaffected members of families with a history of febrile seizures and temporal lobe epilepsy provide support for the hypothesis that preexisting abnormalities may predate the seizure (Fernandez et al., 1998). The third option would be a combination of the above two alternatives, whereby there is a cumulative effect of having a brain-at-risk along with an effect of the seizure itself. Recent animal studies support this view by showing that experiencing seizures during early development while having a brain with malformations of cortical development may worsen cognitive outcomes in the short but not the longer term (Lucas et al., 2011).

Ninety-six percent of PFS cases and 59\% of nonfebrile CSE cases were recruited into this study following their first ever CSE, that is, prior to the potential onset of epilepsy. This enabled us to isolate the effects of CSE from the effects of ongoing seizures and/or medication, especially in the 
case of the PFS group. A different approach with a similar aim was adopted by Adachi et al. (2005), who carried out FSIQ assessments of adult patients with a diagnosis of epilepsy at two time points and compared those who had experienced a CSE in between assessments to those who had not. No differences were observed between the two groups in FSIQ, suggesting that CSE in itself was not the critical factor influencing performance in the context of ongoing epilepsy.

In the current study, we have shown that performance remains unchanged in the first year post CSE. Namely, although the majority of children in our study acquired new skills from baseline to follow-up, their standardized scores were similar at both time points. Moreover, we have demonstrated that seizure-related variables such as duration were not predictive of developmental outcomes. The latter results are in agreement with those of a recent study that found no statistically significant differences in cognitive and motor development between children with febrile seizures lasting a mean of $3.82 \mathrm{~min}$ and those with febrile seizures lasting a mean of $39.82 \mathrm{~min}$ (Hesdorffer et al., 2011). The mean cognitive scores reported within this study were similar to those obtained in our study, even though the mean seizure length of our patients was almost twice as long. Taken together, these findings exclude the theoretical possibility that PFS has a transient effect on performance a few weeks onward. Namely, if seizure activity in itself affects outcome it does so in a more permanent fashion.

It was perhaps not surprising to find that children with previous neurologic deficits, that is, structural abnormalities and prior developmental delay, had the worst developmental outcomes. Past reports investigating outcomes following CSE may have failed to consider the impact of such confounding factors, resulting in an overestimation of the cognitive morbidity attributed to CSE (e.g., Aicardi \& Chevrie, 1970). Therefore, in the future, it may be important to use standardized questionnaires to query developmental functions antedating the seizure to better represent premorbid intellectual abilities.

Age at testing was also identified as an important predictor of performance. However, when we looked at each assessed area in turn, we found that this age dependency stemmed from a relationship between age and language performance in the PFS and the control groups only. Obtaining the same relationship within our control group points away from an insultrelated explanation of this phenomenon. Nevertheless, this is an unexpected finding when using a standardized test, where age has been accounted for in the process of standardization. The lack of a relationship between age and language scores in the higher functioning nonfebrile CSE group argues against an administration and/or test issue. Therefore, it remains plausible that the older children assessed in this study had better language abilities than their younger counterparts. This possibility does not compromise our conclusions, since this observation was made for both the PFS and the control groups.

\section{Limitations}

A possible limitation of this study is that the investigated CSE cohort may not be representative of the population as a whole. Specifically, our cohort contains a lower proportion of children with AS (5.6\% vs. 22.6\%) compared to the nonparticipating cohort. Although this is an important limitation, we believe that it does not compromise the current results. The inclusion of these children possibly would have widened the performance gap between the nonfebrile CSE and the other two groups, as the acute symptomatic group were among the lowest performers. A second potential limitation is that only $35 \%$ of the identified sample agreed to take part in our study. However, participants and nonparticipants did not differ on seizure duration or any demographic variables, suggesting that the participating sample is a good representation of the population as a whole. Moreover, the recruited number of participants makes this study one of the largest in the literature, taking into account that we employed standardized neuropsychological and imaging measures and were able to reassess $70.4 \%$ of our baseline cohort a year onward. Finally, within the PFS group, there was a bigger representation of female patients than would be expected from an epidemiologic perspective (Chin et al., 2006). Nevertheless, there were no gender effects on performance in our group, suggesting that PFS were not differentially affecting girls and boys at this developmental age and, therefore, that the female skew of our sample is not biasing our results.

\section{Conclusions}

In conclusion, the current study has shown decreased levels of functioning in all children following CSE, including those following PFS, who are not normally associated with neurologic abnormalities prior to their episode. The finding that these impairments are still present 1 year post CSE suggests that the seizure itself is not having a transient effect on developmental abilities (i.e., affect performance at 6 weeks but not at 1-year follow-up). However, the present data allow for the possibility that CSE may have a longer lasting impact on future development through a more permanent reorganization of functional networks, which may have already taken place when we first assess these children. Further longitudinal studies incorporating rigorous neurocognitive techniques are required to better understand this issue.

\section{Acknowledgments}

We thank the participants and their families for giving their time so generously to participate in this study. We would also like to thank our local collaborators for helping us with the recruitment phase of our study. We would like to thank the Wellcome Trust for financial support. This work was undertaken at GOSH/UCL Institute of Child Health, which received a proportion of funding from the Department of Health's NIHR Biomedical Research Centres funding scheme. The Centre for Paediatric Epidemiology and Biostatistics also benefits from funding support from the Medical Research Council in its capacity as the MRC Centre of Epidemiology for Child Health. RFMC held a National Institute for Health Research (NIHR) 
Academic Clinical Lectureship. RCS is supported by Great Ormond Street Hospital Children's Charity. We would also like to thank Dr. Suresh Pujar for reading the present manuscript and offering his advice.

\section{Disclosure}

None of the current authors have any conflict of interest to disclose. We confirm that we have read the journal's position on issues involved in ethical publication and affirm that this report is consistent with those guidelines.

\section{REFERENCES}

Adachi N, Kanemoto K, Muramatsu R, Kato M, Akanuma N, Ito M, Kawasaki J, Onuma T. (2005) Intellectual prognosis of status epilepticus in adult epilepsy patients: analysis with Wechsler Adult Intelligence Scale-revised. Epilepsia 46:1502-1509.

Aicardi J, Chevrie JJ. (1970) Convulsive status epilepticus in infants and children. A study of 239 cases. Epilepsia 11:187-197.

Anderson PJ, De Luca CR, Hutchinson E, Roberts G, Doyle LW. (2010) Underestimation of developmental delay by the new Bayley-III scale. Arch Pediatr Adolesc Med 164:352-356.

Baram TZ, Shinnar S. (2001) Do febrile seizures improve memory? Neurology 57:7-8.

Bayley N. (2005) Bayley scales of infant and toddler development-third edition. The Psychological Corporation, San Antonio, TX.

Chang Y-C, Guo N-W, Huang C-C, Wang S-T, Tsai J-J. (2000) Neurocognitive attention and behavior outcome of school-age children with a history of febrile convulsions: a population study. Epilepsia 41:412-420.

Chin RF, Neville BG, Peckham C, Bedford H, Wade A, Scott RC. (2006) Incidence, cause, and short-term outcome of convulsive status epilepticus in childhood: prospective population-based study. Lancet Neurol 368:222-229.

Choy M, Cheung KK, Thomas DL, Gadian DG, Lythgoe MF, Scott RC. (2010) Quantitative MRI predicts status epilepticus-induced hippocampal injury in the lithium-pilocarpine rat model. Epilepsy Res 88:221-230.

Committee on Integrating the Science of Child Development. (2000) From neurons to neighborhoods: the science of child development. National Academy Press, Washington, DC.

Cormack F, Cross JH, Isaacs E, Harkness W, Wright I, Vargha-Khadem F, Baldeweg T. (2007) The development of intellectual abilities in paediatric temporal lobe epilepsy. Epilepsia 48:201-204.

DeLorenzo RJ, Pellock JM, Towne AR, Boggs JG. (1995) Epidemiology of status epilepticus. J Clin Neurophysiol 12:316-325.

Dubé CM, Richichi C, Bender RA, Chung G, Litt B, Baram TZ. (2006) Temporal lobe epilepsy after experimental prolonged febrile seizures: prospective analysis. Brain 129:911-922.

Dubé CM, Zhou JL, Hamamura M, Zhao Q, Ring A, Abrahams J, McIntyre K, Nalcioglu O, Shatskih T, Baram TZ, Holmes GL. (2009) Cognitive dysfunction after experimental febrile seizures. Exp Neurol 215:167-177.

Fernandez G, Effenberg O, Vinz B, Steinlein O, Elger CE, Döhring W, Heinze HJ. (1998) Hippocampal malformation as a cause of familial febrile convulsions and subsequent hippocampal sclerosis. Neurology 50:909-917.

Hesdorffer DC, Benn EKT, Bagiella E, Nordli D, Pellock J, Hinton V, Shinnar S. (2011) Distribution of febrile seizure duration and associations with development. Ann Neurol 70:93-100.

Hesdorffer DC, Shinnar S, Lewis DV, Moshé SL, Nordli DR, Pellock JM, MacFall J, Shinnar RC, Masur D, Frank LM, Epstein LG, Litherland C, Seinfeld S, Bello JA, Chan S, Bagiella E, Sun S; the FEBSTAT Study Team. (2012) Design and phenomenology of the FEBSTAT study. Epilepsia 53:1471-1480.

International League Against Epilepsy, Commission on Epidemiology and Prognosis. (1993) Guidelines for epidemiologic studies on epilepsy. Epilepsia 34:592-596.

Kölfen W, Pehle K, König S. (1998) Is the long-term outcome of children following febrile seizures favourable? Dev Med Child Neurol 40:667-671.

Lucas MM, Lenck-Santini P-P, Holmes GL, Scott RC. (2011) Impaired cognition in rats with cortical dysplasia: additional impact of early life seizures. Brain 134:1684-1693.
Martinos MM, Yoong M, Patil S, Chin RFM, Neville BG, Scott RC, De Haan M. (2012) Recognition memory is impaired in children following prolonged febrile seizures. Brain 135:3153-3164.

Meldrum BS, Brierley JB. (1973) Prolonged epileptic seizures in primates. Ischemic cell change and its relation to ictal physiological events. Arch Neurol 28:10-17.

Nelson KB, Ellenberg JH. (1978) Prognosis of children with febrile seizures. Pediatrics 61:720-727.

Office of the Deputy Prime Minister. (2007) The English Indices of Deprivation 2004: Summary (revised). Office of the Deputy Prime Minister, London.

Raspall-Chaure M, Chin RF, Neville BG, Scott RC. (2006) Outcome of paediatric convulsive status epilepticus: a systematic review. Lancet Neurol 5:769-779.

Ravizza T, Rizzi M, Perego C, Richichi R, Velískǒvá J, Moshé SL, De Simoni MG, Vezzani A. (2005) Inflammatory response and glia activation in developing rat hippocampus after status epilepticus. Epilepsia 46(Suppl. 5):113-117.

Riney CJ, Harding B, Harkness WJ, Scott RC, Cross JH. (2006) Hippocampal sclerosis in children with lesional epilepsy is influenced by age at seizure onset. Epilepsia 47:159-166.

Rutten A, Van Albada M, Silveira DC, Cha BH, Liu X, Hu YN, Cilio MR, Holmes GL. (2002) Memory impairment following status epilepticus in immature rats: time course and environmental effects. Eur J Neurosci 16:501-513.

Sankar R, Shin D, Liu H, Mazarati A, Pereira DV, Wasterlain CG. (1998) Patterns of status epilepticus-induced neuronal injury during development and long-term consequences. J Neurosci 18:8382-8393.

Schiottz-Christensen E, Bruhn P. (1973) Intelligence, behaviour and scholastic achievement subsequent to febrile convulsions: an analysis of discordant twin-pairs. Dev Med Child Neurol 15:565-575.

Scott RC. (2010) Adverse outcomes following convulsive status epilepticus in children: relationship with hippocampal injury. Epilepsia 51(Suppl. 3):178-181.

van Esch A, Ramlal IR, van Steensel-Moll HA, Steyerberg EW, DerksenLubsen G. (1996) Outcome of febrile status epilepticus. Dev Med Child Neurol 38:19-24.

Verity CM, Butler NR, Golding J. (1985) Febrile convulsions in a national cohort followed up from birth. II-Medical history and intellectual ability at 5 years of age. Br Med J (Clin Res Ed) 290:1311-1315.

Verity CM, Greenwood R, Golding J. (1993) Outcome of childhood status epilepticus and lengthy febrile convulsions: findings of a national cohort study. Br Med J 307:225-228.

Verity CM, Greenwood R, Golding J. (1998) Long-term intellectual and behavioural outcomes of children with febrile convulsions. $N$ Engl $J$ Med 338:1723-1728.

Visser AM, Jaddoe VWV, Ghassabian A, Schenk JJ, Verhulst FC, Hofman A, Tiemeier H, Moll HA, Arts WF. (2012) Febrile seizures and behavioural and cognitive outcomes in preschool children: the Generation R study. Dev Med Child Neurol Published online: 03-Sep2012; doi: 10.1111/j.1469-8749.2012.04405.x.

Yoong M, Madari R, Martinos M, Clark C, Chong K, Neville B, Chin R, Scott R. (2012) The role of magnetic resonance imaging in the followup of children with convulsive status epilepticus. Dev Med Child Neurol 54:328-333.

\section{SUPPORTING INFORMATION}

Additional Supporting Information may be found in the online version of this article:

Table S1. Table of developmental delay as per parent report in patients following CSE.

Table S2. Description of MRI abnormalities in the nonfebrile CSE group.

Table S3. List of medications.

Table S4. Cognitive, language, and motor composites for two PFS patients and their healthy twins. 